\title{
Factors affecting saphenous vein graft patency: Clinical and angiographic study in 1402 symptomatic patients operated on between 1977 and 1999
}

\author{
Pallav J. Shah, MBBS, MS, MCh \\ Ian Gordon, MSc, PhD, AStat ${ }^{\mathrm{b}}$ \\ John Fuller, MBBS, FRCP (Edin), FRACPc \\ Siven Seevanayagam, MBBS (Hons), FRACS ${ }^{a}$ \\ Alex Rosalion, MBBS, FRACS ${ }^{a}$ \\ James Tatoulis, MBBS, FRACS ${ }^{c}$ \\ Jai S. Raman, MB, BS, MMed, FRACS, PhD ${ }^{\mathrm{a}}$ \\ Brian F. Buxton, MB, MS, FRACS, FRCS, FRCS(C) ${ }^{\mathrm{a}}$
}

From the Department of Cardiac Surgery, Austin Hospital, ${ }^{a}$ Melbourne, Victoria, Australia, the Statistical Consulting Centre, University of Melbourne, ${ }^{\mathrm{b}}$ Parkville, Victoria, Australia, and Epworth Medical Centre, ${ }^{\mathrm{c}}$ Melbourne, Victoria, Australia.

Received for publication Dec 19, 2002; revisions requested Jan 22, 2003; revisions received May 21, 2003; accepted for publication June 5, 2003.

Address for correspondence: Brian F. Buxton, MB, MS, FRACS, FRCS, FRCS(C), Austin Hospital, Studley Road, Heidelberg, Victoria, 3084 Australia (E-mail: brian. buxton@austin.org.au).

J Thorac Cardiovasc Surg 2003;126:1972-7

Copyright (C) 2003 by The American Association for Thoracic Surgery

$0022-5223 / 2003 \$ 30.00+0$

doi:10.1016/S0022-5223(03)01276-5
Background: The purpose of this study was to find the preoperative and intraoperative factors that affect vein graft patency.

Methods: A total of 3715 graft angiograms in 1607 patients were studied for recurrence of angina. The preoperative patient characteristics and intraoperative variables were prospectively collected from patients who had primary coronary artery bypass grafting during the period from 1977 to 1999 . A total of $1339(83 \%)$ patients were male, with a mean age of 59 years. The mean period from operation to reangiogram was 99 months. The saphenous vein was grafted to the left anterior descending artery in $557(15 \%)$, to the diagonal artery in $669(18 \%)$, to the obtuse marginal artery in $1300(35 \%)$, to the right coronary artery in $409(11 \%)$, and to the posterior descending artery in $780(21 \%)$ cases. Graft failure was defined as $\geq 80 \%$ stenosis.

Results: During the course of the study, 2266 (61\%) grafts were patent, and 1449 $(39 \%)$ had failed. The patient variables that significantly reduced graft patency were a younger age $(P<.001)$ and an ejection fraction $<30 \%(P=.047)$. Operative variables associated with reduced graft patency were small coronary artery diameter $(P<.001)$, large conduit diameter $(P=.001)$, and the coronary artery grafted (lowest patency in the right coronary artery and maximum patency in the left anterior descending artery territory; $P=.002)$. The interval from operation to repeat angiogram $(P<.001$, with $78 \%$ patent at 1 year, $78 \%$ at 5 years, $60 \%$ at 10 years, and $50 \%$ at 15 years) and the year in which the operation was performed (more recent operations had better patency; $P<.001$ ) significantly affected graft patency.

Conclusions: Saphenous vein graft patency improved over the course of the study. The best results were obtained in older patients with good left ventricular function. Large-caliber arteries on the left system, when grafted with a small-diameter vein, were associated with the best outcome.

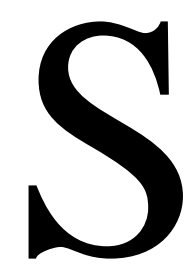

ince the introduction of saphenous vein grafting in the late 1960s, ${ }^{1,2}$ coronary artery bypass grafting, with or without arterial conduits, remains the treatment standard for management of intractable angina due to coronary artery occlusive disease. However, it soon became evident that the saphenous vein provided only palliation of the ongoing process, which is further complicated by vein graft atherosclerosis. Approximately $15 \%$ to $20 \%$ of vein grafts occlude in the first year, and half occlude within the first 2 weeks. Thereafter, the annual occlusion rate is $1 \%$ to $2 \%$ 
from 1 and 6 years and 4\% to 5\% from 6 to 10 years. At 10 years, approximately $60 \%$ of vein grafts are patent; only $50 \%$ of these vein grafts remain free of significant stenosis. ${ }^{3-5}$ The pathology in the vein grafts documented by angiography and histologic examinations includes acute thrombosis and intimal hyperplasia during the first postoperative year and onset of progressive atherosclerosis beyond 3 to 5 years. $3,4,6$

Vein graft failure is one of the primary reasons for coronary bypass reoperations. The economic impact is staggering in an era of intense cost cutting. The fact that some vein grafts remain free of disease for years whereas extensive atherosclerosis develops in others remains an enigma. The purpose of this study was to analyze 25 years of clinical and angiographic data to delineate the patient and operative variables responsible for vein graft failure and, thus, define the patients most susceptible to recurrence of angina and reoperations.

\section{Method \\ Study Population}

The study population included 1607 patients who had undergone a primary coronary artery bypass surgery between 1977 and 1999 with at least 1 vein graft and who later had a recurrence of symptoms that necessitated a coronary angiogram. Patients with a concomitant procedure during the primary operation were excluded.

The preoperative patient characteristics and intraoperative variables were methodically recorded in the database since 1977. A total of 3715 graft angiograms were studied in 1607 patients. Because of missing data, a slightly reduced study population (3218 graft angiograms in 1402 patients) was used to analyze relevant factors affecting graft patency.

The preoperative patient characteristics are described in Table 1. The data on cholesterol levels, incidence of smoking, and peripheral vascular disease were largely missing. The number of saphenous veins grafted to each of the target coronary arteries was as follows: the left anterior descending artery (LAD), 557 (15\%); diagonal, 669 (18\%); obtuse marginal artery, 1300 (35\%); posterior descending artery, $780(21 \%)$; and right coronary artery (RCA), 409 (11\%).

The distribution of intervals from operation to angiogram is described in Figure 1. The maximum number of angiograms was performed between 90 months (7.5 years) and 110 months $(9.2$ years) after surgery. The mean interval from operation to reangiogram was 99 months, or 8.3 years. Aspirin was routinely given in postoperative patients from 1983 and lipid-lowering agents were routinely given from 1994.

\section{Operation}

Coronary artery bypass grafting was performed by a similar protocol by all surgeons. The left internal thoracic artery was used from 1982. All operations were performed on cardiopulmonary bypass with the use of antegrade blood cardioplegia in the early part of the study and with antegrade/retrograde blood cardioplegia in the later part.
TABLE 1. Baseline patient characteristics

\begin{tabular}{lc}
\hline Variable & Data \\
\hline Mean age (y) & 59 \\
Sex (male) & $1339(83 \%)$ \\
NYHA class III or IV & $325(20 \%)$ \\
Diabetes & $161(10 \%)$ \\
Hypertension & $932(58 \%)$ \\
LVEF $<30 \%$ & $55(3.4 \%)$ \\
Urgent operation & $158(9.8 \%)$ \\
\hline
\end{tabular}

NYHA, New York Heart Association; LVEF, left ventricular ejection fraction.

The distal anastomosis to the target coronary artery was constructed end to side or side to side. Proximal anastomosis was almost always to the aorta. In the latter part of the study, especially after 1995, we commenced extensive arterial revascularization. A total of 1456 internal thoracic and 226 radial arterial conduits were used in this group of 1607 patients.

Veins were harvested by a conventional open "no touch" technique. Veins from the calf were generally preferred. Veins were dilated to their naturally distended state, and overdistention was avoided. In the earlier part of the study, veins were dilated and stored in Ringer's lactate, but from 1983, a solution containing Ringer's lactate, blood, papaverine $(1 \mathrm{mmol} / \mathrm{L})$, and heparin $(5000$ $\mathrm{U} / 100 \mathrm{~mL}$ ) was used.

\section{Angiographic Analysis}

The native and graft angiograms were performed in a standard fashion. The left internal thoracic artery and each aortic anastomosis were selectively injected. If the status of vein grafts could not be determined by graft or stump injection, an aortic root angiogram was performed. In patients who had 2 or more postoperative angiograms, the first angiogram was used for analytical purposes. Two cardiologists and a surgeon read the angiograms. The method of reporting was uniform. All the grafts were described in detail, including the degree of stenosis and coronary grafting.

A graft was considered patent when it had less than $80 \%$ stenosis, and the entire course of the graft was visualized, including proximal anastomosis, distal anastomosis, and opacification of the distal target coronary artery. A graft was considered to have failed if it had $80 \%$ stenosis or more or was completely occluded. In sequential vein grafts, each segment was analyzed as a separate graft.

\section{Statistical Analysis}

In the following analysis, a data set was created with key variables that had no missing data. This resulted in a data set of 3218 grafts from 1402 patients. The time of graft failure was usually not recognizable and could have occurred any time between the operation and angiogram. Standard time-related analysis overestimates graft patency. We have therefore analyzed graft patency by using categorical time intervals.

A generalized linear mixed model was fitted to the binary variable, defined as 1 if graft stenosis or occlusion was $80 \%$ or more and 0 otherwise. This is an extension of logistic regression that allows for 2 levels of variation that are present in the data. 


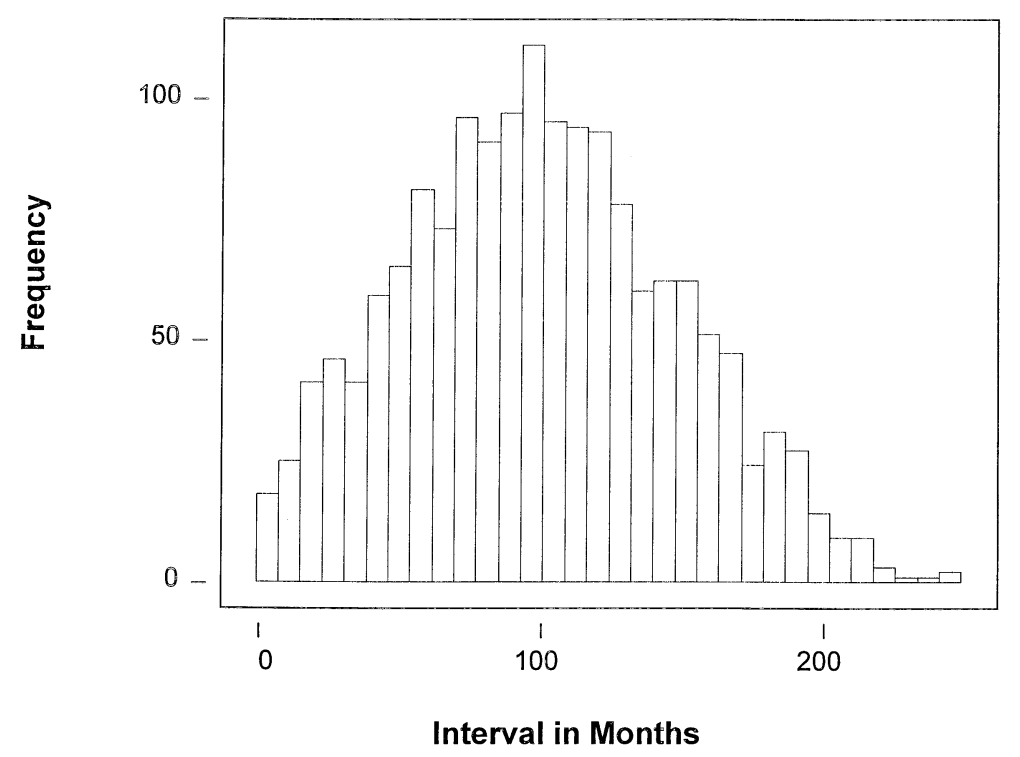

Figure 1. Number of angiograms performed in relation to the time interval after the operation.

Some variables are constant for all grafts from a given patient: for example, age, sex, year of operation, and interval to angiogram. Other variables pertain to individual grafts and therefore take different values for grafts from the same patient, for example, the coronary artery to which the graft is anastomosed. Unlike standard logistic regression, the analysis allows for incorporation of these 2 levels of variation. ${ }^{7}$ The model is multivariate in the usual sense that each variable's effect is adjusted for the effects of other variables considered. The model was fitted by using S-Plus (Insightful Corporation, Seattle, Wash) and the MASS library of additional functions. ${ }^{8}$

\section{Results}

A total of 2267 (61\%) grafts were patent: 1858 (50\%) grafts had $0 \%$ to $19 \%$ stenosis, 106 (2.85\%) grafts had $20 \%$ to $40 \%$ stenosis, $121(3.25 \%)$ grafts had $40 \%$ to $60 \%$ stenosis, and $182(4.9 \%)$ grafts had $60 \%$ to $79 \%$ stenosis. A total of 1449 (39\%) grafts failed: 334 (9\%) grafts had $80 \%$ to $99 \%$ stenosis, whereas 1115 (30\%) grafts were completely occluded. The factors associated with graft patency were analyzed as patient and operative variables; the detailed results are shown in Table 2. With graft failure as the outcome, odds ratios, 95\% confidence intervals, and $P$ values are shown for the patient and operative variables. Some variables were included in the model as continuous, and others were categorical or binary. The odds ratio for a continuous variable corresponds to a difference of a specified number of units. For example, the estimated odds ratio of graft failure for an interval between operation and angiogram of 15 years, compared with 5 years or any other interval of 10 years, was 1.68 (95\% confidence interval, 1.28 to 2.21). For categorical and binary variables, 1 level was chosen as a baseline, and odds ratios for the other levels were expressed in relation to it. For example, the odds ratio of graft failure for the target artery being diagonal, compared with LAD, was 1.29 (95\% confidence interval, 0.99 to 1.68$)$. Finally, the $P$ values shown are tests of significance for each variable separately; in the case of a categorical variable at several levels, the single $P$ value tests the null hypothesis that the risk of graft failure was the same for all levels.

The following list describes the relationship of patient variables to graft patency:

- Younger age $(P<.001)$ and left ventricular ejection fraction $<30 \%(P=.047)$ significantly reduced graft patency. Male sex $(P=.9)$, diabetes $(P=.5)$, hypertension $(P=.5)$, and urgent operation $(P=.9)$ were not significantly associated with graft patency (Table 2).

- Year of operation $(P<.001)$ significantly affected graft patency. For any given angiogram interval, more recently performed operations were associated with better graft patency.

- Interval from operation to angiogram (Figure 2) significantly affected graft patency $(P<.001): 79$ grafts were studied at less than 1 year, 670 grafts between 1 and 4 years, 1603 grafts between 5 and 9 years, 1084 grafts at 10 to 14 years, and 279 grafts at $\geq 15$ years. Graft patency of angiograms studied at less than 1 year, 1 to 4 years, 5 to 9 years, 10 to 14 years, and $\geq 15$ years was $78 \%, 78 \%, 60 \%, 50 \%$, and $50 \%$, respectively. lows:

Operative variables were related to graft patency as fol-

- The target coronary artery grafted $(P=.002)$ significantly affected graft patency. Grafts to the RCA had the worst patency, and those to the LAD had the best 
TABLE 2. Results of a multivariate model: odds ratios (OR) and $95 \%$ confidence intervals (CI) for the associations between patient and operative variables and graft occlusion

\begin{tabular}{|c|c|c|c|c|c|}
\hline Variable & Baseline & Comparison & $\mathbf{O R}$ & $95 \% \mathrm{Cl}$ & $P$ value \\
\hline \multicolumn{6}{|l|}{ Patient variables } \\
\hline Year of operation & $x$ & $x+10$ & 0.37 & $0.27-0.51$ & $<.001$ \\
\hline Age $(y)$ & $x$ & $x+10$ & 0.76 & $0.68-0.86$ & $<.001$ \\
\hline Interval between operation and angiogram (y) & $x$ & $x+10$ & 1.68 & $1.28-2.21$ & $<.001$ \\
\hline Sex & Female & Male & 1.02 & $0.78-1.34$ & .9 \\
\hline Hypertension & No & Yes & 1.07 & $0.88-1.31$ & .5 \\
\hline Diabetes & No & Yes & 1.12 & $0.81-1.55$ & .5 \\
\hline Low ejection fraction $(<30 \%)$ & No & Yes & 0.59 & $0.36-0.99$ & .047 \\
\hline Urgent operation & No & Yes & 0.99 & $0.71-1.39$ & .9 \\
\hline NYHA class III or IV & No & Yes & 0.96 & $0.69-1.32$ & .8 \\
\hline \multicolumn{6}{|l|}{ Operative variables } \\
\hline \multirow[t]{4}{*}{ Target artery } & LAD & Diagonal & 1.29 & $0.99-1.68$ & .002 \\
\hline & & Marginal & 1.39 & $1.11-1.75$ & \\
\hline & & Right & 1.63 & $1.22-2.17$ & \\
\hline & & $\begin{array}{c}\text { PDA or LV } \\
\mathrm{Br}\end{array}$ & 1.34 & $1.03-1.74$ & \\
\hline \multirow[t]{5}{*}{ Target artery diameter (mm) } & $0-0.9$ & $1.0-1.4$ & 1.59 & $0.44-5.79$ & $<.001$ \\
\hline & & $1.5-1.9$ & 0.93 & $0.26-3.35$ & \\
\hline & & $2.0-2.4$ & 0.78 & $0.22-2.82$ & \\
\hline & & $2.5-3.4$ & 0.61 & $0.16-2.30$ & \\
\hline & & Endarterectomy & 1.69 & $0.27-10.50$ & \\
\hline \multirow[t]{4}{*}{ Target artery stenosis } & $1 \%-39 \%$ & $40 \%-59 \%$ & 1.53 & $0.66-3.54$ & .18 \\
\hline & & $60 \%-79 \%$ & 1.18 & $0.52-2.65$ & \\
\hline & & $80 \%-99 \%$ & 1.08 & $0.48-2.42$ & \\
\hline & & Total & 1.28 & $0.57-2.88$ & \\
\hline \multirow[t]{4}{*}{ Conduit diameter (mm) } & $1.5-2.4$ & $2.5-3.4$ & 1.09 & $0.43-2.77$ & .001 \\
\hline & & $3.5-4.4$ & 1.62 & $0.64-4.11$ & \\
\hline & & $4.5-5.4$ & 2.17 & $0.83-5.67$ & \\
\hline & & $\geq 5.5$ & 2.36 & $0.77-7.16$ & \\
\hline \multirow[t]{2}{*}{ Conduit wall thickness } & Thin & Normal & 1.29 & $1.04-1.59$ & .07 \\
\hline & & Thick & 8.04 & $1.20-53.78$ & \\
\hline Distal anastomosis & End to side & Side to side & 1.01 & $0.63-1.62$ & $>.9$ \\
\hline
\end{tabular}

$P D A$, Posterior descending artery; NYHA, New York Heart Association; $L V B r$, left ventricular branch.

patency. The order of increasing patency was RCA $\rightarrow$ obtuse marginal artery $\rightarrow$ posterior descending artery $\rightarrow$ diagonal $\rightarrow$ LAD (Table 2 ).

- Target coronary artery diameter $(P<.001)$ significantly affected graft patency. The worst to best graft patency was endarterectomy $\rightarrow 0$ to $0.9 \mathrm{~mm} \rightarrow 1.0$ to 1.4 $\mathrm{mm} \rightarrow 1.5$ to $1.9 \mathrm{~mm} \rightarrow 2$ to $2.4 \mathrm{~mm} \rightarrow 2.5$ to $3.4 \mathrm{~mm}$.

- Conduit diameter $(P=.001)$ significantly affected graft patency. Large-diameter veins were associated with worst graft patency; better graft patency was associated with smaller conduit size. The worst to best graft patency was $\geq 5.5 \mathrm{~mm} \rightarrow 4.5$ to $5.4 \mathrm{~mm} \rightarrow 3.5$ to $4.4 \mathrm{~mm} \rightarrow 2.5$ to $3.4 \mathrm{~mm} \rightarrow 2$ to $2.4 \mathrm{~mm}$.

- Conduit wall thickness $(P=.07)$ showed a trend toward affecting graft patency. The conduit wall thickness was graded as thick, normal, and thin veins. Thick wall was defined as $>1.5 \mathrm{~mm}$, normal as 1.0 to 1.5 $\mathrm{mm}$, and thin as $<1.0 \mathrm{~mm}$. Only 7 conduits were described as thin. Thick-walled veins were associated with worst graft patency.
- Target coronary artery stenosis $(P=.18)$ and distal anastomosis type, that is, end to side or side to side (sequential), were not significantly associated with graft patency $(P>.9)$.

\section{Discussion}

This is one of the largest reported angiographic studies ${ }^{4}$ of saphenous vein graft patency. The study was performed over 25 years and analyzed clinical and operative variables. The results confirm that $20 \%$ of the grafts occlude within the first year of surgery. After the first year, the annual occlusion rate is $2 \%$ to $5 \%$. At 10 to 14 years, $50 \%$ of the grafts were patent, $30 \%$ were completely occluded, and $20 \%$ showed varying degrees of atherosclerotic changes. This proportion was similar at 15 years and beyond.

Thrombosis and neointimal hyperplasia are considered to be the cause of occlusion in the first year, and subsequently atherosclerosis begins after 3 years. ${ }^{3,4,6}$ The cause of early occlusion is probably related to the surgical technique of harvesting and storing the saphenous vein and the precision 


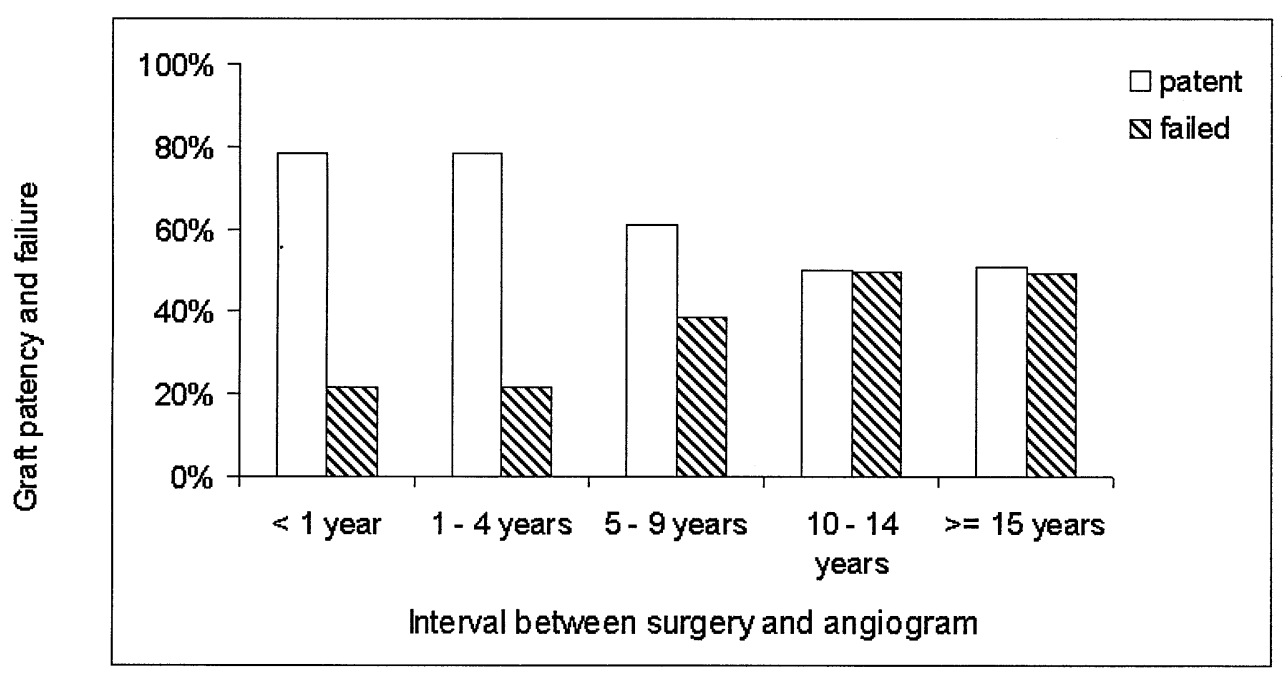

Figure 2. Graft patency at $<1$ year, 1 to 4 years, 5 to 9 years, and 10 to 14 years after the primary operation.

of anastomosis. ${ }^{6,9}$ The precise cause of late atherosclerosis remains unknown, although many theories exist and may provide avenues for improvement in vein graft patency. ${ }^{6}$ Recently, to overcome these shortcomings, arterial conduits are used with increasing frequency. The internal thoracic artery does not usually develop late postoperative atherosclerosis, and its patency at 10 years is approximately $90 \% .{ }^{10}$ However, because of the relative ease with which veins can be harvested and anastomosed, the saphenous vein remains widely used, and improvement in their long-term patency remains a major objective.

In our study, more recent operations were associated with better graft patency. This could be due to routine use of aspirin, vigorous treatment with cholesterol-lowering agents, and better harvesting, preparation, and storing techniques of vein grafts that have evolved over the last 3 decades. Aspirin $(325 \mathrm{mg} / \mathrm{d})$ is associated with improved saphenous vein graft patency during the first year. ${ }^{11}$ The post-coronary bypass graft trial ${ }^{12}$ showed that aggressive decreasing of low-density lipoprotein cholesterol to $<100$ $\mathrm{mg} / \mathrm{dL}$ decreased obstructive changes in saphenous vein grafts by $31 \%$. Harvesting, preparation, and storing techniques significantly affect graft patency. ${ }^{6,9,13}$

Younger age and a lower ejection fraction independently reduced vein graft patency, unlike in other studies. ${ }^{13,14} \mathrm{~A}$ possible explanation is that younger patients have higher prevalence of risk factors (variables such as smoking and cholesterol, which were not analyzed in our study) and more severe coronary disease. Reduced ejection fraction may indicate large areas of infarcted myocardium with poor distal runoff. In our study, variables such as hypertension, sex, diabetes, and myocardial infarction did not affect graft patency, similar to other reports. ${ }^{13,14}$
Severity of coronary artery stenosis ${ }^{15}$ and type of distal anastomosis $^{13,16}$ did not affect graft patency in our study, unlike other reports. In this study, $91 \%$ of the veins were grafted to vessels with $\geq 60 \%$ stenosis. There was no significant difference in the graft patency when the target artery was totally occluded or had a stenosis of $60 \%$ to $79 \%$ or $80 \%$ to $99 \%$. Veins, unlike arterial grafts, are less susceptible to spasm and are less affected by competitive flow and autoregulation. Target arteries with $>70 \%$ stenosis are grafted. A significant association was found between target coronary artery diameter and graft patency, similar to other reports. ${ }^{13,17}$ The most likely reason is that a large-diameter vessel has a better runoff and, therefore, better graft patency.

The saphenous vein conduit diameter and wall thickness significantly affected graft patency, similar to the results of earlier series. ${ }^{13,17}$ Poor results that followed the use of large-diameter and thick-walled saphenous veins may be the result of low-velocity flow within the conduit leading to deposition of oxidized low-density lipoprotein in the graft wall. The thigh vein is often harvested because of convenience and the mistaken belief that bigger is better. Indeed, the saphenous vein is often harvested by the endoscopic technique or with the aid of a vein stripper, which are better suited to above-knee use. This study conclusively identifies that larger and thicker-walled veins that are typically harvested from the thigh are associated with poor long-term patency.

In this study, a novel statistical analysis was used. Because some variables are unique to an individual patient and are constant for all grafts for that patient, whereas other variables are unique to a graft and vary with the patient, it is invalid to use ordinary logistic regression. To put it another way, to do so would be to treat the data set of 3218 
grafts as if it came from 3218 patients, each with a single graft, when in fact there were 1402 patients with 3218 grafts. This data set violates the assumption of independence between observations that is required for the use of ordinary logistic regression. The graft stenosis outcomes for grafts from the same individual are not independent from one another. The method used is a newly available approach that accounts properly for the 2 levels of variation and allows valid inferences to be drawn. ${ }^{7}$

Our current practice is to use arterial grafts. However, when required, veins are harvested from the calf because they are a better size match to the coronary arteries. Veins of normal wall thickness are preferred. The saphenous vein is suitable for grafting vessels with intermediate to severe stenosis. The best results are obtained when veins are grafted to the left system.

Our study had several limitations. The graft failure event was assumed to have occurred at the time of the angiogram, but the graft could have actually blocked at any time from the date of the operation to the date of the angiogram. Our patient population included those who had symptom-directed angiography, and this is only a small proportion $(15 \%)$ of all patients who underwent surgery during that time. Some of the patient variables known to affect graft patency were not analyzed because of missing data. The measurements for operative variables are based on visual assessment and may vary from surgeon to surgeon. Finally, different calendar periods may be associated with use of different methods of harvesting, preparation, and anastomotic techniques.

\section{Conclusion}

Saphenous vein graft patency improved with time. The best results were obtained in older patients with good left ventricular function. Large-caliber arteries on the left system, when grafted with a small-diameter vein, were associated with the best outcome.

\section{References}

1. Garrett HE, Dennis EW, DeBakey ME. Aortocoronary bypass with saphenous vein graft. Seven-year follow-up. JAMA. 1973;223:792-4.
2. Favaloro RG. Saphenous vein graft in the surgical treatment of coronary artery disease: operative technique. J Thorac Cardiovasc Surg. 1969;58:178-5.

3. Bourassa MG. Fate of venous grafts: the past, the present, and the future. J Am Coll Cardiol. 1991;5:1081-3.

4. Fitzgibbon GM, Kafka HP, Leach AJ, Keon WJ, Hooper D, Burton JR. Coronary bypass fate and patient outcome: angiographic follow up of 5,065 grafts related to survival and reoperation in 1,388 patients during 25 years. J Am Coll Cardiol. 1996;28:616-26.

5. Campeau L, Enjalbert M, Leasperance J, Bourassa MG, Kwiterovich $\mathrm{P}$ Jr, Wacholder S, et al. The relation of risk factors to the development of atherosclerosis in saphenous vein bypass grafts and the progression of disease in native circulation: a study 10 years after aortocoronary bypass surgery. $N$ Engl J Med. 1984;311:1329-32.

6. Shuhaiber JH, Evans AN, Massad MG, Geha AS. Mechanisms and future directions for preventions of vein graft failure in coronary bypass surgery. Eur J Cardiothorac Surg. 2002;22:387-96.

7. Breslow NE, Clayton DG. Approximate inference in generalized linear mixed models. J Am Stat Assoc. 1993;88:9-25.

8. Venables WN, Ripley BD. Modern applied statistics with S. 4th ed. New York: Springer; 2002. p. 292-8.

9. Campeau L, Crochet D, Lesperance J, Bourassa MG, Grondin CM. Postoperative changes in aortocoronary saphenous vein grafts revisited: angiographic studies at 2 weeks and at 1 year in two series of consecutive patients. Circulation. 1975;52:369-77.

10. Carrier M. The internal mammary artery: why is it the graft of choice?. In: Carrier M, Pelletier LC, editors. Conduits for myocardial revascularization. Austin (TX): RG Landes Co; 1993. p. 34-58.

11. Goldman S, Copeland J, Moritz T, Henderson W, Zadina K, Ovitt T, et al. Saphenous vein graft patency 1 year after coronary artery bypass surgery and effects of antiplatelet therapy. Results of a Veterans Administration Cooperative Study. Circulation. 1989;80:1190-7.

12. The effect of aggressive lowering of low-density lipoprotein cholesterol levels and low-dose anticoagulation on obstructive changes in saphenous-vein coronary-artery bypass grafts. The Post Coronary Artery Bypass Graft Trial Investigators. N Engl J Med. 1997;336:153-62.

13. Souza DS, Dashwood MR, Tusi JCS, Filbey D, Bodin L, Johansson B, et al. Improved patency in vein grafts harvested with surrounding tissue: results of a randomized study using three harvesting techniques. Ann Thorac Surg. 2002;73:1189-95.

14. Korpilahti K, Engblom E, Syvänne M, Hamalainen H, Puukka P, Vanttinen E, et al. Angiographic changes in saphenous vein grafts and atherosclerosis risk factors. A 5-year study with serial measurements of serum lipids and lipoproteins. Scand Cardiovasc J. 1998;32:343-51.

15. Roth JA, Cukingnan RA, Brown BG, Gocka E, Carey JS. Factors influencing patency of saphenous vein grafts. Ann Thorac Surg. 1979; 28:176-83.

16. Flemma RJ, Johnson WD, Lepley D Jr. Triple aorto-coronary vein bypass as treatment for coronary insufficiency. Ann Thorac Surg. 1971;103:82-3.

17. Björk VO, Ekeström S, Henze A, Ivert T, Landou C. Early and late patency of aortocoronary vein grafts. Scand J Thorac Cardiovasc Surg. 1981;15:11-21. 